\title{
Atrial Tachycardia: A Rare Type of Supra Ventricular Tachycardia
}

\author{
M Aziz, M Ullah, M Hossain, MA Ali \\ Dept. of Cardiology, NICVD, Dhaka
}

\begin{abstract}
Key Words :
Atrial

Atrial tachycardia is a relatively uncommon form of SVT. Atrial tachycardia can be observed in persons with normal heart and in those with congenital heart disease and particularly after surgery for repair or correction of congenital or valvular heart disease. Atrial tachycardia tends to be refractory to pharmacologic therapy and is therefore frequently treated with ablative therapy. If untreated, it may lead to tachycardia-induced cardiomyopathy. So, atrial tachycardia needs to be discussed for recognition and appropriate management. We have gone through different journals to review the latest informations about atrial tachycardia.
\end{abstract}

Abstract:

(Cardiovasc. j. 2012; 4(2): 164-170)

\section{Introduction:}

Any supravertricular tachycardia (SVT) that requires only atrial tissue for its initiation and maintenance can properly be considered as atrial tachyarrhythmia. ${ }^{1}$ Atrial tachycardia can be defined as supravertricular tachycardia that does not require atrioventricular junction, accessory pathways or ventricular tissue for initiation and maintenance of tachycardia. ${ }^{2}$ In 2001 the joint expert group from the working group of arrhythmias of European Society of Cardiology and North American Society of Pacing and electro physiology (Now called Heart Rhythm Society) classified regular atrial tachycardia according electro physiologic mechanism and anatomy. The Joint expert group defined "Focal atrial tachycardia as being characterized by atrial activation starting rhythmically at a small area (focus) from where it spreads antrifugally". ${ }^{3}$ This definition indicates that arrhythmia arises from an area that is smaller than would be required for classical macro reentry. Atrial tachycardia can be observed in persons with normal heart and in those with congenital heart disease and particularly after surgery for repair or correction of congenital or valvular heart disease. ${ }^{4}$ Although a relatively uncommon form of SVT, atrial tachycardia tends to refractory to pharmacologic therapy and is therefore frequently treated with ablative therapy.
Atrial fibrillation and Atrial flutter although fulfilling this definition of atrial tachycardia and typically identified as specific entities. Atrial tachycardia is a subset of supraventricular tachycardia, rate of which is variable ranges from $100-250 \mathrm{~b} / \mathrm{min}^{4}{ }^{4}$

Atrial tachycardia most commonly is seen in children with congenital heart disease in whom cardiac surgery has been performed. It also occurs in children with cardiac surgery has or congenital heart disease and may result in significant morbidity. ${ }^{4}$

\section{Epidimiology}

Atrial tachycardia is relatively rare constituting $5 \%-15 \%$ of all supraventricular tachycardia. ${ }^{4}$ Foetal arrhythmias occur in approximately in $1-2 \%$ of pregnancy of which 5-8\% due to non-sinus tachycardia. In children and infants without cardiac surgery primary atrial tachycardia appears throughout the infancy and childhood and accounts for $14 \%$ of supraventricular cases. ${ }^{5}$ Based estimated prevalence of SVT of 1 in 200 to 400 children the prevalence of primary atrial tachycardia in children is approximately 0.03 to 0.08 percent . By comparison ectopic atrial tachycardia in adult males was 0.34 percent. $^{6}$

\section{Anatomy}

Atrial tachycardia can have either right or left atrial origin. Some atrial tachycardia usually originate 
outside the usual anatomic boundaries of the atrial such as pulmonary venous ostia. ${ }^{7,8} \mathrm{Al}$ though atrial tachycardia tends to originate along the crista terminalis, atrial appendages, tricuspid annulus. Other sites are possible these sites are common for automaticity or triggered activity. ${ }^{9,10}$ Additionally disease processes or age related degeneration of atria may give rise to arrhythmogenic substrate. In 1997, Prasantha et al. reported about a new entity of adenosine sensitive atrial tachycardia probably due to focal reentry within the atrioventricular nodal transitional area but this unusual site. Other uncommon sites of focal atrial conduction are those originating superior venacava. ${ }^{11}$

\section{Pathophysiology}

Several pathologic mechanisms have been ascribed to atrial tachycardia. These mechanism scan be differentiated based on their electrophysiological mechanism. These are enhance \& automaticity, triggered or micro reentrant mechanism.

\section{Enhanced Automaticity}

Automatic Atrial tachycardia is observed both in patients with normal heart structure and is those with organic heart disease. Tachycardia typically exhibits a warm up phenomenon during which atrial rate gradually accelerate after its initiation and slows prior to termination. ${ }^{12}$ It is rarely initiated or termination by a single atrial stimulation or rapid atrial pacing, but it may be transiently suppressed by overdrive pacing. Carotid sinus massage and adenosines do not terminate this-tachycardia if they produce a transient atrioventricular block. Electric cardioversion is ineffective. ${ }^{11}$

\section{Triggered Activity}

Triggered activity is due to delayed after depolarization which are low amplitude oscillations occurring at the end of action potential. If these oscillations are of sufficient amplitude to reach threshold potential, depolarization occurs again and spontaneously action potential is generated. If single it is atrial ectopic. If it recurs and spontaneous depolarization continues a sustained tachycardia may result. This can be induced with rapid atrial pacing example is digitalis intoxication, characteristically this arrhythmia can be initiated, accelerated, and terminated by rapid atrial pacing but no entrainment, tachycardia initiation is cycle length dependent. ${ }^{12}$ It may be sensitive to physiologic and pharmacologic maneuver such as adenosine, verapamil and beta blockers. ${ }^{11}$

Reentrant tachycardia is two types a) Macro reentry b) Micro reentry.

a) Macro reentry - is usual mechanism in atrial flutter and in scar and incision related (Post surgical) atrial tachycardia. The more common and recognized form atrial tachycardia seen with advent of pulmonary vein isolation and linear ablation procedures is left atrial tachycardia. These tachycardia may be self limiting. 8,13

b) Micro reentry- can arise in a small focal area such as sinus node reentry. Reentry tachycardia arises suddenly, terminates suddenly, and is paroxymal. $8,11,13$

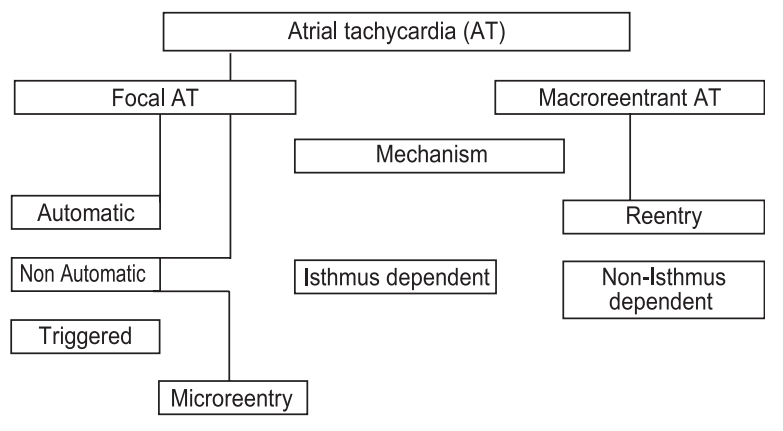

Fig.-1: Mechanisms \& classification of Atrial Tachycardia ${ }^{14}$

\section{Classification}

Atrial tachycardia can be classified according to endocardial activation mapping data, pathophysiological mechanisms and anatomy. ${ }^{15}$

Ø Focal atrial tachycardia (due to automatic, triggered or microrentry mechanism)

$\varnothing$ Macroreentrant atrial tachycardia (including isthmus dependent atrial flutter and others originating from right or left.

$\varnothing$ Multifocal atrial tachycardia (MAT)- is a rare arrhythmia in childhood and in often related to underline heart or lung disease. ${ }^{3}$

\section{Aetiology}

Atrial tachycardia can occur with normal structural heart disease or in patients with organic heart 
Table I

Characteristics of different mechanism of Focal Atrial Tachycardia.

\begin{tabular}{lccc}
\hline & Automatic AT & Triggered Activity & Microreentry \\
\hline PES & No Response & CL dependent & Reproducibly initiated and \\
terminates AT & & & \\
Isoprenalin for induction & Yes & No & No \\
Entrainment & No & No & Yes \\
Warm up cool down & Yes & No & No \\
Response to Adenosine & No & Yes & Yes \\
Response to Propranolol, Verapamil & Propranolol & Propranolol & Verapamil \\
Vagal Maneuver & No response & Terminates & No Response \\
\hline
\end{tabular}

disease. ${ }^{16,17,18,19}$ It arises in patients with congenital heart disease or who have undergono corrective or palliative cardiac surgery such as Fortan operation. ${ }^{3}$

Atrial tachycardia may manifest during exercise, acute illness, alcohol ingestion, altered fluid state, hypoxia and metabolic disturbances or drug use. Digitalis intoxication is one of the causes being the underlying mechanism. ${ }^{20}$

Multifocal atrial tachycardia is often related to underlying illness. MAT can occur in COPD, valvular heart disease, Diabetes mellitus, Hypokalaemia, Hypo magnesemia, Azotemia, Post operative states, Sepsis. ${ }^{21,22,23,24,25}$

Unusual forms of atrial tachycardia can be seen in patients with infiltrative process involving the pericardium and, by extension, the arterial wall.

Incessant Atrial Tachycardia Resulting In Cardiomyopathy

The term incessant is applied to an Atrial tachycardia when atrial tachycardia is present for at least ninety percent of the time a patient is monitored. ${ }^{2}$

Incessant atrial tachycardia is often found in other wise normal young individuals including children although it may occur with organic heart disease. ${ }^{17,18,19,26}$ The rate tends to be faster during the day than at night and it may increase with exercise or pregnancy.

In whom the rhythm is incessant may develop atrial tachycardia induced cardiomyopathy. ${ }^{26}$ In case reports and small series of patient with atrial tachycardia induced cardiomyopathy frequently have complete resolution of left ventricular dysfunction with successful radiofrequency catheter ablation of atrial focus. ${ }^{27,28}$

\section{Clinical Feature:}

Patient with focal atrial tachycardia usually present with episodic or paroxysmal tachycardia Typically atrial tachycardia manifests as a sudden onset of palpitation. If atrial tachycardia due to enhanced automaticity it may be non-sustained but repetitive or continuous or sustained as in reentrant forms of atrial tachycardia. ${ }^{11}$

Patient may present with tachycardia that gradually speeds up soon after its onset (Warm up phenomenon). ${ }^{12}$ Patient may report dyspnoea, dizziness, light headedness fatigue or chest pressure. Patient may have symptom of underlying disease.

The primary abnormality noted upon physical examination is rapid pulse rate. In most situations it is regular. However in multifocal atrial tachycardia pulse may be irregular. Blood pressure may be low in patients presenting with fatigue, light-headedness or presyncope. The cardiovascular examination should be aimed at excluding heart diseases such as valvular heart disease and evidence of tachycardia induced cardiomyopathy. ${ }^{29}$

\section{Diagnosis}

a) Electrocardiographic feature:

ECG features of atrial tachycardia include $P$ wave morphology and axis, PR interval, P-P interval variation. Typically, an isoelectric line is seen between consecutive $\mathrm{P}$ waves, while no line is seen with macro reentrant arrhythmia (eg. Atrial flutter). ${ }^{3}$ 
In most cases PR interval is shorter than $R P$ interval. In the presence of preexisting A-V nodal conduction delay, PR interval may be longer than RP interval.

Before planning electro physiology study $\mathrm{P}$ wave morphology is very helpful to guide electrophysiological procedure. The most helpful ECG leads are $\mathrm{aVL}$ and $\mathrm{V}$, in distinguishing right from left atrial focus. ${ }^{30,31,32,33}$
A positive or biphasic $\mathrm{P}$ wave in aVL is most likely associated with a RA focus (sensitivity $88 \%$ and specificity $79 \%) .{ }^{30}$

A positive $\mathrm{P}$ wave in $\mathrm{V}$, is closely associated with a LA focus (sensitivity $93 \%$ and specificity $88 \%$ ). ${ }^{30}$ Right atrium - A focus high in crista terminalis has upright $\mathrm{P}$ waves, foci at inferior crista terminalis have inverted $\mathrm{P}$ wave in II, III, aVF ${ }^{31}$ deep negative $\mathrm{P}$ waves in inferior leads and is suggest tricuspid ring. ${ }^{34}$

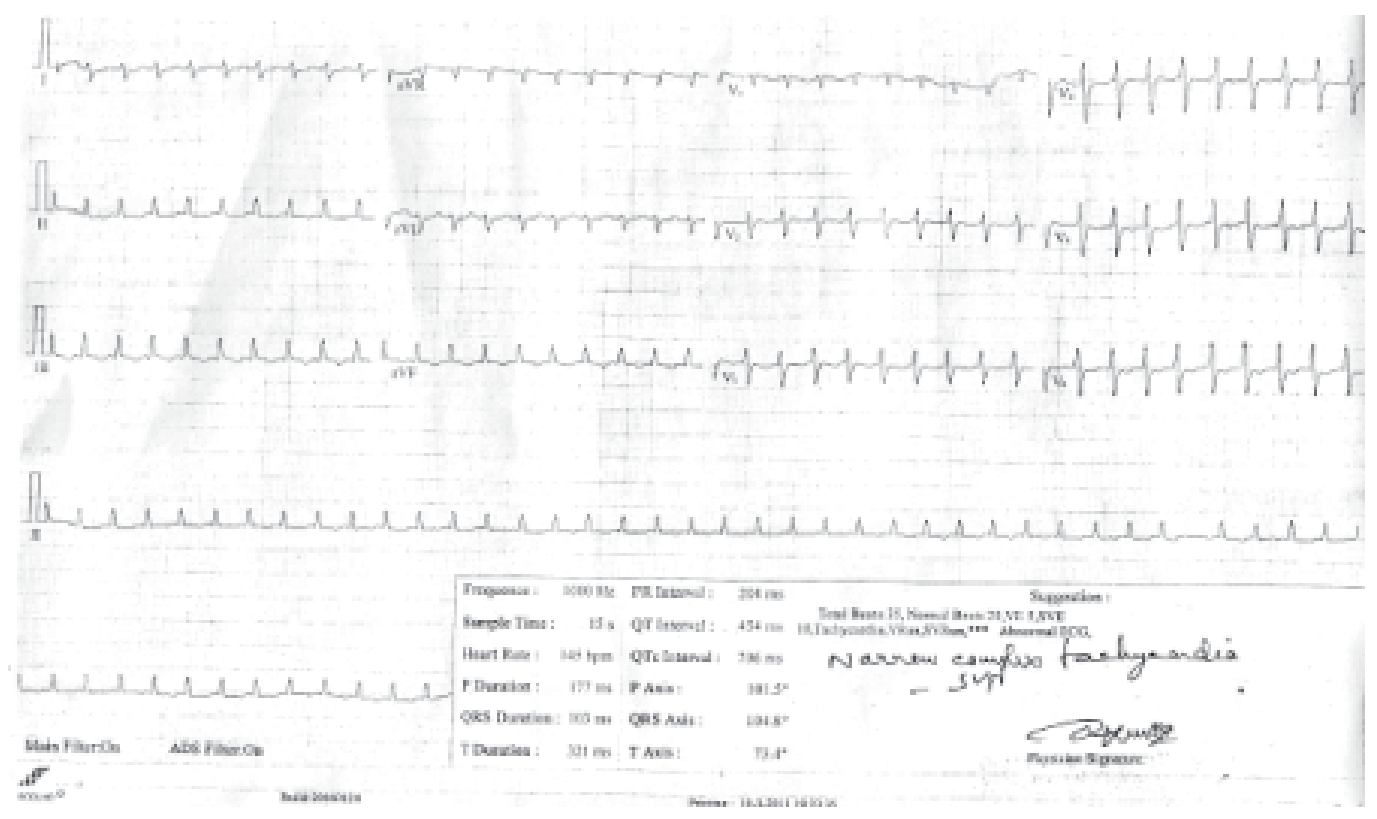

Fig 2: ECG showing Atrial tachycardia.

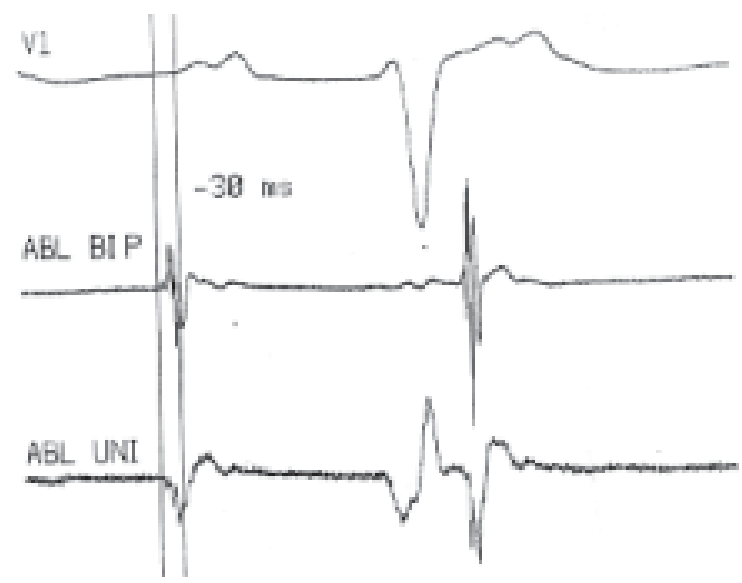

Fig 3: Intracardiac Eletrocardiograms and surface ECG recorded during mapping of the tachycardia. The ablation catheter is positioned transseptally and located at the site where successful ablation was achieved. Local atrial activation precedes the onset of the $P$ wave (vertical line) by $30 \mathrm{~ms}$. The unipolar recording shows a $Q S$ configuration at this site. ( $A B L$ bip = bipolar recording on ablation catheter; $A B L$ uni = unipolar recording on ablation catheter $)$ 
A negative $\mathrm{P}$ wave in inferior leads and positive $\mathrm{P}$ wave in $\mathrm{V}$, coronary sinus origin. ${ }^{12}$

Left atrium - The most common site of origin of left focal atrial tachycardia are the pulmonary veins and mitral annulus. ${ }^{35,36}$

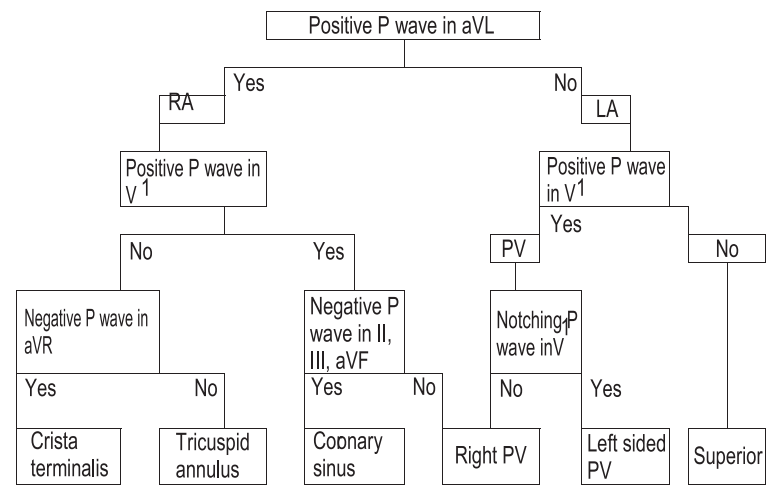

Other investigations are a) Serum electrolytes, b) Blood haemoglobin level and RBC counts to see evidences of anaemia, c) Arterial blood gas analysis to define pulmonary status, d) Serum digoxin.

Treatment -

I. Medical

II. EPS and RFA

I. Anti arrhythmic drug therapy -

Anti arrhythmic drug can prevent recurrences and may be required. ${ }^{37} \mathrm{~A}$ calcium channel blocker or beta blocker may be appropriate as well as in combination therapy for patients without cardiac failure ACC/AHA/ESC 2003 guide line states that class Ia and class Ic agents may be used, for patients with poor ventricular function intravenous amiodarone is preferable.

\section{Drugs in Atrial Tachycardia}

\begin{tabular}{ll}
\hline $\begin{array}{l}\text { Atrial tachycardia } \\
\text { due to }\end{array}$ & Drugs required \\
\hline a. Triggered activity & $\begin{array}{l}\text { Sensitive to verapamil, } \\
\text { beta blockers and } \\
\text { adenosine }\end{array}$ \\
b. Enhanced automatically & Beta blocker \\
c. Refractory recurrent & $\begin{array}{l}\text { Quinidine, } \\
\text { Procainamide or } \\
\text { Flecanide. (Flecaride is } \\
\text { preferable) }\end{array}$ \\
\hline
\end{tabular}

Treatment for maintenance of sinus rhythm Class III anti arrhythmic drug such as amiodarone, sotalol, and dofetelide highly effective for maintaining sinus rhythm after cardioversion to a normal sinus rhythm.

Cardioversion -

For any patient in whom the rhythm is not tolerated well haemodymically and in whom rate control drugs are ineffective or contraindicated cardioversion should be considered. The 2003 ACC/ AHA/ESC guideline is in agreement. Automatic atrial tachycardia and multifocal atrial tachycardia (MAT) do not respond to cardioversion.

\section{Electro physiology Study And Radio Frequency Ablation}

Indications- In some patients, atrial tachycardia is incessant \& cause dilated cardiomyopathy known as a tachycardia mediated cardiomyopathy. ${ }^{29}$ Due to cardiomyopathy incessant atrial tachycardia frequently does not respond well to pharmacologic therapy; as a result patient with tachycardia induced cardiomyopathy should have their tachyarrhythmia treated aggressively with catheter ablation. The 2003 ACC/AHA/ESC guidelines for the management of supraventricular arrhythmias recommended catheter ablation in the following setting. ${ }^{20}$

1) Recurrent Symptomatic Atrial Tachycardia

2) Incessant atrial tachycardia, regardless of symptoms.

\section{Techniques for Catheter Ablation}

Mapping and ablating atrial tachycardia is technically more challenging than other forms of paroxysmal supraventricular tachycardia. ${ }^{38}$ Following electrophysiological criteria are used to make a diagnosis of focal Atrial Tachycardia-

a) $\mathrm{P}$ wave configuration different from that of sinus rhythm (for the clarification of $\mathrm{P}$ wave morphology brief ventricular pacing or intravenous adenosine may be helpful). ${ }^{12}$

b) Occurrence of tachycardia independent of atrial this interval. ${ }^{12}$

c) Spontaneous termination in the absence of Ablock. ${ }^{12}$

d) When burst ventricular pacing accelerates atrial rate and VAAV response is seen after.

e) Termination ventricular pacing, this strongly predicts AT. This is highly sensitive and 
specific and virtually diagnostic of atrial tachycardia. ${ }^{39}$

f) In case of VA conduction, the atrial activation sequence is different from that of tachycardia.

g) VA dissociation during ventricular pacing - The inability to capture the atrial retrogradely during on going tachycardia had a positive predictive value of $80 \%$ for atrial tachycardia. ${ }^{39}$

\section{Catheter Ablation}

Catheter ablation of atrial tachycardia largely depends upon atrial activation mapping to identify an endocardial atrial site that is activated earlier than any other site. Timing is commonly referenced to the surface $\mathrm{P}$ wave. Successful ablation site is typically activated $30 \mathrm{~ms}$ or more prior to onset surfaces $\mathrm{P}$ wave. Other mapping techniques, including pace mapping during sinus rhythm and entrainment mapping during tachycardia are occasionally employed.

\section{Prognosis}

In patients with structurally normal heart, atrial tachycardia is associated with a low mortality rate. However tachycardia induced cardiomyopathy has been associated in patients in whom the rhythm is incessant. In case reports and small series of patients atrial tachycardia induced cardiomyopathy frequently have complete resolution of left ventricular dysfunction with successful radio frequency ablation of atrial focus. ${ }^{27,28} \mathrm{ACC} / \mathrm{AHA} /$ ESC 2003 Guideline cites the high success rate (86\%) and low recurrence rate (8\%) shown by pooled data from 514 patients who had catheter ablation for focal atrial tachycardia. ${ }^{20}$

\section{Conclusion:}

Atrial tachycardia is a rare type of SVT. It is often drug resistant. Atrial tachycardia is often incessant and can produce tachycardia-induced cardiomyopathy. But this cardiomyopathy can be prevented and reversed by radio frequency ablation of the arrhythmia.

\section{References:}

1. Krummen DE, P Mitul, Nguyen H, Ho G, Kazi DS, Clopton P, Holland MC, Greenberg SL, Feld GK, Faddis MN, Narayan SM. Accurate ECG Diagnosis of Atrial Tachyarrhythmias Using Quantitative Analysis: A Prospective Diagnostic and Cost-Effectiveness Study. J Cardiovasc Electrophysiol 2010; 21(11): 1251-1259.
2. Moracky F. Radio frequency ablation as a treatment for cardiac arrhythmia. N Eng J Med 1999; 6: 13061310.

3. Saudi N, Cosio F, Waldo A, Chen SA, Lesaka Y, Lesh M, Saksena S, Salerio J, Schoels W. Classification of Atrial flutter \& regular AT according to electrophysiology mechanism \& anatomic base: a statenad from a joint expert group from the working group of arrhythmias of the European society of pacing \& electrophysiology. J. Cardiovac Electrophysiol 2000;12: 852-866.

4. Sung RJ. Incessant supraventricular tachycardia. Pacing Electrophysiology 1983; 6 : 1306 - 1310.

5. Ko JK, Deal NJ, Stras Burger JF, Benson DW Jr. Supraventricular tachycardia mechanism \& their age distribution in paediatric patients. Am J Cardiol 1992; 69:1028.

6. Poutiainen AM, Kasten MJ, Alrakinen KE, et al. Prevalence and natural course of ectopic atrial tachycardia. EU Heart J 1999; 20:694 -700.

7. Kistler PM, Sanders P, Fynn SP, et al. Electrophysiological and electrocardiographic characteristics of focal atrial tachycardia originating from pulmonary veins: acute and long term outcomes of radiofrequency ablation. Circulation 2003; 108:1968 - 1975.

8. Yamada T, Murakami Y, Muto M et al. Electrophysilogy characteristics of atrial tachycardia originating from the of pulmonary veins or post right atrium: double potentials obtained from the post, wall of right atrium can be useful to predict foci of AT in rtpul veins or post RA. J Cardiovac Electrophysiol 2004; 15:745 -751.

9. Lesh MD, Van Hare GF, Epstein LM et al. Padiofrequency catheter ablation of atrial arrhythmias. Results \& mechanism. Circulation 1994; 89:1074 - 1081.

10. Kalman JM, Olgin JE, Karch MR et al. "Crystal tachycardia"; origin of patient atrial tachycardia from the terminals identified by intracardiac echocardiography. J Am Coll Cardiol 1998; 31: 451457.

11. Characterization of Focal Atrial Tachycardia Using HighDensity Mapping. Prashanthan Sanders, Mélèze Hocini, Pierre Jaïs, Li-Fern Hsu, Yoshihide Takahashi, Martin Rotter, Christophe Scavée,Jean-Luc Pasquié, Fréderic Sacher, Thomas Rostock, Chrishan J. Nalliah, Jacques Clémenty, and Michel Haïssaguerre. J Am Coll Cardiol 2005; 46: 2088 - 2099.

12. Chen SA, Cluang CE, Yang CJ, Cheng CC, WU TJ, Wang SP, Cheang BV, Chang MS 1994. Sustained atrial tachycardia in Adult patient electrophysiological characteristics, Pharmacological response, Possible mechanism \& effects of radiofrequency ablation. Circulation 1990: 1262-1278.

13. Schmitt h, Weber S, Schwab JO, Voss RM, Kneller R, Tillmanns H, Waldecker B. Diagnosis and ablation of focal right atrial tachycardia using a new high resolution, 
non- contact mapping system. Am J Cardiol 2001; 87: $1017-1021$.

14. Donnerstein RL, Berg RA, Shehab Z, Ovadia M. Complex atrial tachycardias and respiratory syncytial virus infection in infant. J Pediatr 1994; 125: 23 - 32.

15. Narula OS. Sinus node reentry: a mechanism for supraventricular tachycardia. Circulation 1974; 50: 1114 -1120 .

16. Gillette PC, Smith RT, Gason A JR, et al. Chronic Supraventricular tachycardia. A curable cause of congestive cardiomyopathy. JAMA 1985; 253: 391 - 399.

17. Gasona Jr et al. Treatment of Atrial automatic tachycardia by ablation procedure. $J$ Am Coll Cardiol 1985; 6: $405-410$.

18. Packer DL, Bardy GH, Worley SJ, et al. Tachycardia Induced cardiomyopathy: a reversible form of left ventricular dysfunction. Am J Cardiol 1986; 57: 563 569 .

19. Bertil Olson S, Blomtron P, Sabel KG, William Olson G. Incessant ectopic atrial tachycardia: Surgical treatment with regression of Dilated Cardiomyopathy picture. Am J Cardiol 1984; 53 : 1465 - 1472.

20. Guidelines for clinical intracardiac electrophysiological and catheter Ablation procedure. A report of American College of Cardiology/American Heart Association task force on practia guidelines (Committe on clinical intracardiac Electrophysiologic \& catheter Ablation procedure). Developed in collaboration with North American Society of Pacing and Electrophysiology. Circulation 1995; 92:678 -690.

21. Tang CW, Scheiman MM, Van Hare GF, Epstein LM, Fitzapatrick AP, Lee RJ, Lesh MD: Use of P wave configaration during atrial tachycardia to predict the origin. J Am Coll Cardiol 1995; 26:1315-1324.

22. Farooki ZQ, Green EW, Multifocal atrial tachycardia in two neonates. Br. Heart J 1977; 39:872- 880.

23. Salim MA, Case CL, Gillette PC. Chatic atrial tachycardia is children. Am Heart $J$ 1995; 129:831 - 839.

24. Fish FA, Metha AV, Johns JA. Characteristics and management chastic atrial tachycardia of infancy. Am J Cardiol 1996; 78: 1052 - 1053.

25. Bradley DJ, Fisch Bach PS, Law I et al. The clinical course of multifocal atrial tachycardia in infants and children. J Am Coll Cardiol 2001; 38:401 - 408.

26. Kistler PM, Rofert Thomson KC, Haqqani HM, Fynn SP, Singarayar S, Vohra JK, Morten JB, Parks PO, Kalman JM: P wave morphology is focal atrial tachycardia, development of an algorithm to predict anatomic site of origin. J Am Coll Cardiol 2006; 48:10101017.
27. Chiladakis JA, Vassilikos VP, Maunis TN, et al. Successful radiofrequency catheter ablation of automatic atrial tachycardia with regression of cardiomyopathy picture. Pacing Clin Electrophysiol 1997; 20: $953-960$.

28. Gillette PC, Wampler PG, Goason A Jr, et al. Treatment of atrial automatic tachycardia by ablation procedures. J Am Coll Cardiol 1985; 6:405 -409.

29. Fish FA, Mehta AV, Jshns JA, Characteristics \& management of chaotic atrial tachycardia of Infancy. Am J cardiol 1996; 78:1052 - 1058.

30. Kistter PM, Kalman JM. Locating focal atrial tachycardias from P wave morphology. Heart Rhythm 2003; 2: 561-564.

31. Tada H, Nogami A, Natio S, Suguta M, Nakatsu gawa M, Horie Y, Tomita T, Hoshizaki H, Oshima S, Taniguchi K. Simple electrocardiographic criteria for identifying the site of origin of focal right atrial tachycardia. Pacing Clin Electrophysiol 1998; 21: 2431-2439.

32. Yamane T, Shah DC, Peng JT, Hecini M, Diesenhofer I, Choi KJ, Maela L, Clementry J, Haissaguerre M. Morphological characteristics of $\mathrm{P}$ wave during selective pul vein pacing. J Am Coll Cardiol 2001; 38: 1505-1510.

33. Tang CW, Scheinman MM, Vein Hare GF, Epstein LM, Fitzpatrick AP, Lee RJ, Lesh MD. Use of P wave configuration during atrial tachycardia to predict site of origin. J Am Coll Cardiol 1995 26: 1315-1324.

34. Morton JB, Sanders P, Das A, Vohra TK, Sparks PB, Kalman JM. Focal atrial tachycardia arising from tricuspid annulus: electrophysiologic \& electrocardio graphic characterstics. J Cardiovase Electrophysiol 2001; 12:653-659.

35. Dong J, Zrenner B, Schrick J, Diesenhofer I, Karch M, Schneider M, Ven Barry C, Weyerbrsck S, Yin Y, Schmitt C. Catheter ablation of left atrial focal tachycardia guided by electroanatomic mapping and new insight in to intra atrial electrical conduction. Heart Rhythm 2005; 2: 598-591.

36. Hachiya H, Ernst S, Quyang F, Mavarakis H, Chan J, Bansch D, Antz M, Kuck KH . Topographic distribution of focal left atrial tachycardias defined by electro cardiographic \& electrophysiological dota, Circulation 2005; 69:205-210.

37. Morady F. Radio-frequency ablation astreatment for cardiac arrhythmia. N,Engl J Med 1999; 340:534 -339.

38. Lseh MD. Radiofrequency catheter ablation of atrial tachycardia and flutter. In: Zipes; DP, Jalife J. Eds. Cardiac electrophysiology from cell to bedside. Philadelphia, WB saunders, 1995, 1461 - 1469.

39. Roberts-Thom Son KC, Kister PM, Kalman JM. Atrial tachycardia mechanisms, diagnosis, and management. Curr Probi Cardiol 1995; 30: 529-573 